\title{
Problemática y beneficios de las actividades acuáticas en la escuela. Problematic and benefits of aquatic activities by school.
}

\author{
Apolonia Albarracín Pérez \\ Universidad de Almeria \\ Juan Antonio Moreno Murcia
}

Universidad Miguel Hernández de Elche

\section{Resumen}

El principal objetivo del estudio ha consistido en comprobar la valoración de las actividades acuáticas por parte del profesorado de educación física en Educación Secundaria, para lo que se ha creado y validado el Cuestionario de Actividades Acuáticas en Educación Física (CAÁEF). También se han comprobado los efectos principales y de interacción que han producido el género, la titulación, la situación laboral, la experiencia docente, el tipo de cursos realizados, quién impartió lás clases de actividades acuáticas y la opinión de aspectos generales de educación física sobre la inclusión de las actividades acuáticas en el ámbito escolar. Los participantes fueron 176 profesores de ESO de la Región de Murcia. Para comprobar la validez del CAAEF se realizó un análisis factorial exploratorio obteniendo dos factores denominados "beneficios de la inclusión" y "problemática del profesorado", con coeficientes de fiabilidad aceptables, y con una varianza total explicada del $35.98 \%$. Tras los respéctivos análisis de varianza se comprobó que las mujeres consideraban más importante los beneficios que acarreaban el incluir dichas actividades en el currículum que los problemas de las mismas. Mientras, la problemática que éstas presentan ha sido más tenida en cuenta por aquellos docentes que tuvieron que impartir las clases con un técnico acuático al ser incluidas en el currículo escolar. El poseer la titulación de Licenciado en Ciencias de la Actividad Física y del Deporte no implicaba un mayor reconocimiento de los beneficios ni una reducción en la aparición de problemas al considerar estas actividades. Los resultados son discutidos en referencia a otras investigaciones y amplia bibliografía.

Palabras clave: actividades acuáticas; educación física; medición; profesorado.

\begin{abstract}
The main objective of the study was to verify the assessment of the aquatic activities in reference to the physical education professors in secondary education for those that participated in the Aquatic Activities in Physical Education Questionnaire (CAAFE). It also tells the main effects and which interactions produced gender, educational background, working status, teaching experience, type of courses completed, who taught the aquatic/water activity classes and the opinion of the general aspects of physical education about the inclusion of water activities in the school. The participants in the survey were one hundred and seventy-six ESO teachers from the Murcia Region. An exploratory factorial analysis used "benefits of inclusion" and "problems of the teachers" as the two dominant factors along with acceptable coefficients. An explained total variance of $35.98 \%$ was used to check the validity of the questionnaire. After the respective variance analysis, it was verified that women considered the benefits from activities in the curriculum more important than their own problems. Whereas, the problems presented were taken in account by the teachers who had to teach classes with a water expert present to be included in the school curriculum. Having a Bachelor of Science degree in Physical Education and Sport does not imply a better knowledge of the benefits nor that fewer problems with the activities will occur. The results were argued among other research and a wide range of bibliography.
\end{abstract}

Key words: aquatic activities; physical education; survey; teachers staff.

Correspondencia/correspondence:Juan Antonio Moreno Murcia

Universidad Miguel Hernández de Elche

Edificio Torrevaillo, Avenida de la Universidad, s/n, 03202 Elche (Alicante)

E-mail: j.moreno@umh.es 


\section{Introducción}

$\mathrm{E}$ 1 entorno de aprendizaje influye en la conducta de las personas convirtiéndose en un valioso instrumento a disposición del profesorado (Blández, 1996). Uno de los entornos que activa el proceso de enseñanza-aprendizaje y dirige al alumnado al descubrimiento de nuevos aprendizajes es el medio acuático y éste nos sitúa ante un ambiente único, ya que inmersos en él se cumplen todas las funciones socialmente atribuidas al movimiento como agente educativo. Según la argumentación de Pérez (1998) un niño puede hacer en el agua todo lo que hace fuera de ella, pero es necesario que se le brinde el entorno propicio para desarrollar sus potenciales. De este modo, el primer paso para estas actividades sería el convencimiento de la justificación educativa de este medio, y a partir de allí adaptar los objetivos, metodologías, contenidos, etc., no cayendo en el error del "todo vale si es dentro del agua" (Albarracín, 2003). Así pues, convencidos de este gran potencial del medio acuático para el desarrollo de la motricidad humana, este puede convertirse en un entorno de aprendizaje educativo.

En este sentido, Vaca $(2000,26)$ considera la piscina como un pequeño microsistema en el que se producen intercambios experienciales entre los propios alumnos, que a la vez se cruzan con las orientaciones educativas del profesor que trata de lograr que cada niño adquiera un sentimiento responsable del medio acuático. Además, ello conllevará la posibilidad de utilizar el medio acuático dentro de la actividad física para el tiempo libre, de forma individual y como promoción de la salud (Wigmore, 1994, 1995, 1997; Williams, Bedward, y Woodhouse, 2000; Zwozdiak-Myers, 2004). Por esta causa, encontramos diferentes opiniones (Boixeda, 2003; Le Boulch, 1991) que consideran que dicha potencialidad se encuentra en estrecha relación con la aplicación de actividades físicas acuáticas dirigidas y organizadas en la etapa de escolarización, destacando las posibilidades formativas para favorecer la experimentación de acciones diferentes y vivencias nuevas, enriqueciendo así el bagaje motriz del alumnado y permitiendo una nueva percepción de este entorno.

Esta reflexión nos aboca hacia la justificación de estas actividades en el ámbito escolar, y más concretamente dentro del área de educación física. En este sentido camina Terret (1998) al plantear una disertación sobre el agua, el espacio y la escuela, afirmando que todo espacio escolar está planificado para que los estudiantes logren una actitud favorable hacia el trabajo, con calidad y economía, restringiendo los espacios inútiles o no funcionales. Así, el medio acuático se ha ido adaptando y convirtiendo en uno de esos espacios educativos que cumplen normas diferentes a los convencionales como gimnasio o aula, pero compartiendo funciones similares a éstos.

Con todo ello consideramos necesario incluir las actividades acuáticas en el currículum escolar, desde la educación infantil hasta la educación física de Enseñanza Primaria y Secundaria, ya que siguiendo a Miró (1984) si dicha programación quiere ser completa debe incluir medio terrestre, aéreo y acuático. Así pues, la motricidad acuática, considerada como un subconjunto de la motricidad terrestre (Del Castillo, 2001), puede desarrollarse como lo hace ésta, necesitando para ello coordinar el nivel de maduración y las suficientes experiencias. En cualquier caso, lo que no parece haber duda es que el trabajo en el medio acuático puede contribuir a la formación personal, ya que en él se desarrolla la capacidad de desenvolverse con cierta autonomía e independencia en un medio no habitual, por lo que se considera idóneo para el desarrollo de los contenidos 
del área de educación física, además de necesario para ser trabajado en la edad escolar (Casterad, 2003; Lacasa y López, 2002; López y Aznar, 2003).

En la actualidad se atisba un cierto auge de las actividades acuáticas debido a los esfuerzos realizados por aquellos que empezaron a tratar la inclusión de las actividades acuáticas en educación física hace más de quince años (Navarro, 1990), convencidos de los beneficios que proporcionaría a esta materia (Joven, 2001b). A pesar de eso, todavía en el momento actual existe problemática al respecto, por no tratarse de una situación común ni generalizada en los centros educativos (López y Aznar, 2003). De hecho, no encontramos referencias legales obligatorias para este contenido en todo el período considerado dentro de la Reforma Educativa de la LOGSE ni en los primeros Reales Decretos. Será con los nuevos decretos autonómicos, en los que aparecerá en algún caso ciertas referencias, pero sólo aludiendo a este medio de forma muy general y escueta (D. 112/2002, D.113/2002, D.291/2007 y D.262/2008).

Además de la ausencia de referencias legales, la realidad de las prácticas acuáticas tanto en Enseñanza Primaria como en Secundaria es que éstas no están implantadas de forma habitual dentro de las programaciones escolares, y prácticamente sólo aparecen programas municipales gracias a la iniciativa de los propios profesionales de educación física. De hecho, así se concluye en un estudio realizado a todos los currículos de educación física en Enseñanza Primaria de las diferentes administraciones educativas, referido a la situación de las actividades acuáticas en tales documentos legales (Jofre y Lizalde, 2003).

Por estas razones, existen condicionantes a la hora de abordar la educación física en el medio acuático, pudiendo señalar de forma general como problemas o dificultades para llevar a cabo un programa de actividades acuáticas en el ámbito escolar aspectos como (Albarracín, 2005, 2007; Barter y Firth, 1994; Gourson- Verger y Verger, 2003; Hardman, 2001; Joven, 1990, 2001a; Lawton y Lee, 1995; Pichot, 2006; Tuero y Salguero, 2003): la instalación, el tiempo empleado para desplazamientos y cambios, la formación de los docentes o técnicos acuáticos, los programas, el coste de la actividad, la escuela y los padres. Por su parte, Lacasa y López (2002) dividen estos obstáculos para la inclusión curricular en dos puntos determinantes: los condicionantes intrínsecos del sistema educativo vigente $\mathrm{y}$ el grado de accesibilidad a las instalaciones $\mathrm{y}$ equipamientos acuáticos.

En cuanto al primer punto, con la LOGSE se ofreció un papel activo al profesorado a la hora de elaborar el currículo, por lo que para transmitir todos los tipos de actividades físicas que existen se han visto obligados a priorizar unos contenidos sobre otros, por lo que estos autores creen, que a pesar de que las actividades acuáticas sean una parte importante del currículo motor del alumnado, no son habitualmente tratadas en las programaciones, ya que dan prioridad a otros contenidos. Por otro lado, Joven (2001a) coloca al docente como principal responsable para incluir estas actividades, y sin embargo, en torno al profesional de las actividades acuáticas en horario escolar, existen dudas acerca de quién debe ser el responsable de impartir las clases, siendo variada la posición de los diferentes autores, aunque en definitiva se puede resumir en tres posturas: que sean impartidas exclusivamente por el profesor, únicamente por el técnico acuático de la instalación, o una colaboración entre ambos (Rédaction EPS, 2003; Casterad, 2004; González et al., 2003). Respecto del segundo punto, la disponibilidad de instalaciones es uno de los grandes impedimentos que se presentan con estos planteamientos. 
Así pues, el objetivo principal del trabajo ha consistido en comprobar la valoración del profesorado de educación física acerca de la problemática y beneficios que puede presentar la inclusión de las actividades acuáticas en su materia, para lo que se ha creado un nuevo instrumento de medida. Los objetivos secundarios van encaminados a valorar la importancia de la formación tanto inicial como continua (titulación y cursos realizados) en la consideración de las actividades acuáticas en la escuela, en función de los beneficios y problemas presentados. De igual forma, se ha analizado en qué medida la valoración de aspectos generales de la educación física se relacionan con la opinión de estas actividades, así como otros aspectos tanto personales (género y edad) y laborales de los docentes (experiencia docente, situación administrativa, etc.), como de la propia práctica acuática (personal que ha impartido las clases).

Como hipótesis se ha planteado que la valoración de determinados aspectos de la educación física se van a relacionar con la consideración de los beneficios y problemas que conlleva la inclusión de las actividades acuáticas en dicha materia. Por otra parte, una mayor experiencia docente, una titulación acorde con la materia, una situación administrativa más estable, así como cursos de especialización en estos contenidos favorecerán el aumento de la consideración de los beneficios y una reducción de las dificultades encontradas. Por último, el género no supondrá una mayor consideración ni de los beneficios ni de la problemática de las actividades acuáticas.

\section{Material y Método}

\section{Participantes}

La muestra estuvo compuesta por 176 docentes del área de educación física (129 hombres y 47 mujeres), de una población de 240, con edades comprendidas entre los 21 y los 60 años $(M=36.63, D T=9.67)$, pertenecientes a los centros de Enseñanza Obligatoria de la Región de Murcia.

\section{Instrumentos}

Se han utilizado dos escalas en esta investigación, las cuales han sido creadas para este caso, apoyándose para su construcción en otros instrumentos (Benito, 1995; Moreno, 1998).

Cuestionario de Actividades Acuáticas en Educación Física (CAAEF). Mide las opiniones de los docentes de educación física hacia la aplicación dentro del horario lectivo de contenidos acuáticos a través de 17 ítems, agrupados en 2 factores: beneficios de la inclusión (e.g. "los beneficios físicos y psíquicos que proporciona el medio acuático por sus características especiales son mayores que en el medio terrestre" y problemática para el profesorado (e.g. "muchos profesores de educación física desconocen las posibles aplicaciones de las actividades acuáticas en su materia").

Valoración de Educación Física (VEF). Mide las opiniones de los docentes de educación física hacia aspectos relevantes de dicha materia que pueden influir decisivamente en contenidos poco tradicionales, como es el caso de las actividades acuáticas. Está compuesto de 12 ítems, agrupados en un solo factor (e.g. "los profesores de educación física no imparten aquellos contenidos que no saben o no controlan" o "los centros educativos no están acondicionados para una educación física de calidad").

En los dos casos se mide a través de ítems policotómicos en una escala tipo Likert de 5 puntos donde el 1 corresponde a totalmente en desacuerdo y 5 a totalmente de acuerdo. 
Variables socio-demográficas. En el cuestionario se incluyeron variables como el género, edad, situación administrativa, titulación y experiencia docente, así como otros aspectos que implican la actualización y formación permanente en el centro.

\section{Procedimiento}

Tras la revisión de diferentes instrumentos, se establecieron dos dimensiones hipotéticas de estudio en el CAAEF y una dimensión en el VEF. Posteriormente se sometió la batería de ítems a una evaluación por parte de expertos en la temática (Lynn, 1986). Los mismos estimaron que los ítems fueron pertinentes para medir el constructo que se creó, además de la correcta redacción de los mismos. Antes de pasar la escala a la muestra definitiva, se administró, en primer lugar, a una muestra compuesta por 20 participantes (Osterdind, 1989), observándose que algunas palabras y expresiones presentaban problemas de comprensión. Éstas fueron corregidas y finalmente se administró el cuestionario a toda la población (totalidad de docentes de educación física de la Región de Murcia), aunque al final la muestra se redujo a un elevado porcentaje de la misma $(80 \%)^{1}$. En la administración final se informó a los participantes a través del cuestionario acerca de cómo cumplimentar el mismo, insistiendo en que no dejaran ninguna pregunta sin contestar, así como el anonimato de sus respuestas, siendo la participación totalmente voluntaria. La administración fue realizada directamente, por correo postal así como por correo electrónico. El tiempo aproximado para cumplimentar el cuestionario fue de 15 minutos.

\section{Análisis de datos}

En primer lugar para obtener la estructura factorial de CAAEF y VEF, se realizó un análisis factorial exploratorio, y a continuación un análisis de consistencia interna a través del alfa de Cronbach. Después se realizó un análisis descriptivo y de correlación entre todas las variables. Para obtener los resultados sobre los efectos principales y de interacción entre las distintas variables se realizó un MANOVA. El programa estadístico utilizado para el análisis fue el SPSS 15.0.

\section{Resultados}

\section{Propiedades psicométricas del CAAEF y VEF}

Análisis factorial exploratorio (AFE) del CAAEF. Por la falta de evidencias previas de una correlación entre los factores con la muestra descrita se realizó un análisis factorial exploratorio de los componentes principales con rotación varimax. Tras un primer análisis se descartaron los ítems 7 ("Las actividades acuáticas no se introducen habitualmente en las programaciones de educación física por falta de instalaciones"), 16 ("Me parecería adecuada una legislación que obligase a impartir actividades acuáticas a ciertos niveles, facilitando el tema de la instalación y seguridad") y 17 ("En otros países la legislación educativa facilita ciertos contenidos como esquí, natación, orientación, etc., y se imparten de forma cotidiana dentro del currículo ordinario"), al no alcanzar la saturación mínima de .40 (Stevens, 1992). Con los 14 ítems restantes se volvió a realizar un AFE (Tabla 1), quedando agrupados en dos factores denominados: beneficios de la inclusión y problemática del profesorado, con autovalores por encima de 1.00 (4.35 y 1.76, respectivamente), y una varianza total explicada del $35.98 \%$.

\footnotetext{
${ }^{1}$ De los 220 docentes de educación física en la Región de Murcia, según datos ofrecidos por la Consejería de Educación, participaron en la investigación 176.
} 
Albarracín, A.; Moreno, J. A. (2009). Problemática y beneficios de las actividades acuáticas en la escuela. Revista Internacional de Ciencias del Deporte, 16(5), 71-89. http://www.cafyd.com/REVISTA/01605.pdf

Tabla 1. Análisis Factorial Exploratorio del CAAEF.

\begin{tabular}{|c|c|c|}
\hline Factores & 1 & 2 \\
\hline \multicolumn{3}{|l|}{ Beneficios } \\
\hline $\begin{array}{l}\text { 2. Los beneficios físicos y psíquicos que proporciona el medio acuático por } \\
\text { sus características especiales son mayores que en el medio terrestre }\end{array}$ & .545 & \\
\hline $\begin{array}{l}\text { 3. La actividad física en el medio acuático tiene menos peligro para las } \\
\text { lesiones (menos impacto, más relajación) por lo que es muy beneficiosa }\end{array}$ & .496 & \\
\hline $\begin{array}{l}\text { 4. Todos los bloques de contenido en educación física son susceptibles de } \\
\text { ser impartidos en el agua, con la adecuación suficiente }\end{array}$ & .444 & \\
\hline $\begin{array}{l}\text { 5. El desarrollo motriz en el niño se ve favorecido por una experiencia } \\
\text { precoz en el medio acuático }\end{array}$ & .607 & \\
\hline $\begin{array}{l}\text { 6. Es importante que todos los niños en edad escolar sepan nadar para } \\
\text { evitar accidentes }\end{array}$ & .520 & \\
\hline $\begin{array}{l}\text { 9. La natación es un deporte conocido, practicado y beneficioso como para } \\
\text { ser incluido en las clases de educación física }\end{array}$ & .533 & \\
\hline $\begin{array}{l}\text { 10. La natación es el deporte más completo y el más prescrito por los } \\
\text { médicos }\end{array}$ & .613 & \\
\hline $\begin{array}{l}\text { 11. En una clase de educación física en el agua conseguiríamos la } \\
\text { participación de los alumnos exentos por problemas de espalda }\end{array}$ & .436 & \\
\hline $\begin{array}{l}\text { 12. El medio acuático es atractivo, motivante y novedoso, y el aspecto } \\
\text { recreativo lo tenemos asegurado }\end{array}$ & .644 & \\
\hline $\begin{array}{l}\text { 13. Muchos alumnos sólo han asistido a la piscina para bañarse, y } \\
\text { desconocen otras posibles actuaciones }\end{array}$ & .349 & \\
\hline \multicolumn{3}{|l|}{ Problemática del profesorado } \\
\hline $\begin{array}{l}\text { 1. El término "actividades acuáticas educativas" es un gran desconocido } \\
\text { por el profesorado de educación física }\end{array}$ & & .683 \\
\hline $\begin{array}{l}\text { 8. Las actividades acuáticas no se introducen habitualmente en las } \\
\text { programaciones de educación física porque el profesorado no está formado } \\
\text { para ello }\end{array}$ & & .631 \\
\hline $\begin{array}{l}\text { 14. Mucho profesorado de educación física desconoce las posibles } \\
\text { aplicaciones de las actividades acuáticas en su materia }\end{array}$ & & .820 \\
\hline $\begin{array}{l}\text { 15. Mucho profesorado de educación física, aún teniendo posibilidad de ir a } \\
\text { piscinas, no lo hacen por comodidad y seguridad personal }\end{array}$ & & .765 \\
\hline Autovalores & 4.35 & 1.76 \\
\hline \% varianza & 25.62 & 10.36 \\
\hline \% varianza total & & 35.98 \\
\hline
\end{tabular}

Análisis de consistencia interna del CAAEF. La consistencia interna del instrumento se obtuvo calculando el coeficiente alfa de Cronbach. El factor 1 (beneficios de la inclsuión) estaba compuesto por diez ítems, y se refiere a los aspectos positivos de la incorporación de las actividades acuáticas en las programaciones educativas de educación física. Incluye ítems tales como "Todos los bloques de contenido son susceptibles de ser impartidos en el agua, con la adecuación suficiente", "Es importante que todos los niños en edad escolar sepan nadar para evitar accidentes", o "El medio acuático es atractivo, motivante y novedoso, y el aspecto recreativo está asegurado". La consistencia interna de este factor fue de .73. 
El factor 2 (problemática del profesorado) lo componían 4 ítems, referidos a las dificultades del profesorado hacia la aplicación de las actividades acuáticas en su práctica docente. Lo conformaban ítems como: "Mucho profesorado de educación física desconoce las posibles aplicaciones de las actividades acuáticas en su materia" o "Mucho profesorado de educación física, aún teniendo la posibilidad de ir a piscinas, no lo hacen por comodidad y seguridad personal". La consistencia interna fue de .74.

Análisis factorial exploratorio del VEF. Tras un AFE se obtuvo un único factor denominado valoración de la educación física, con un autovalor de 3.62, y una varianza total explicada de $13.93 \%$ (Tabla 2 ).

Tabla 2. Análisis Factorial Exploratorio del VEF.

\begin{tabular}{|c|c|}
\hline ítems & 1 \\
\hline $\begin{array}{l}\text { 1.Creo que los compañeros de otras materias no valoran demasiado la educación } \\
\text { física }\end{array}$ & .607 \\
\hline $\begin{array}{l}\text { 2.En general, los padres están convencidos de que la educación física no tiene } \\
\text { entidad propia }\end{array}$ & .635 \\
\hline $\begin{array}{l}\text { 3.Aún hoy, los trabajos más laboriosos es sensibilizar a los padres sobre la } \\
\text { importancia de la educación física }\end{array}$ & .610 \\
\hline $\begin{array}{l}\text { 4.Los centros educativos no están acondicionados para ofrecer una educación física } \\
\text { de calidad }\end{array}$ & .531 \\
\hline $\begin{array}{l}\text { 5.Para educar como pretende a ley, el profesorado debería tener un máximo de } 20 \\
\text { alumnos por grupo }\end{array}$ & .511 \\
\hline $\begin{array}{l}\text { 6. Me preocupa no disponer de conocimientos y estrategias que orienten mi práctica } \\
\text { educativa hacia la legislación educativa actual }\end{array}$ & .333 \\
\hline $\begin{array}{l}\text { 7.La educación física sigue siendo considerada como una asignatura } \\
\text { complementaria }\end{array}$ & .618 \\
\hline 8.La educación física debería tener mayor peso específico en el currículo & .588 \\
\hline $\begin{array}{l}\text { 9.Dos horas semanales de educación física no son suficientes para cumplir los } \\
\text { objetivos que marca la legislación educativa }\end{array}$ & .490 \\
\hline $\begin{array}{l}\text { 10.Los profesores de educación física no imparten aquellos contenidos que no } \\
\text { saben o no controlan }\end{array}$ & .315 \\
\hline $\begin{array}{l}\text { 11.Es importante cumplir las prescripciones de la ley acerca de aumentar el } \\
\text { número de experiencias motrices }\end{array}$ & .340 \\
\hline $\begin{array}{l}\text { 12.En educación física se consideran peligrosas ciertas innovaciones curriculares, } \\
\text { por lo que no se llevan a cabo }\end{array}$ & .317 \\
\hline Autovalores & 3.62 \\
\hline \% varianza & 13.93 \\
\hline
\end{tabular}

Análisis de consistencia interna del VEF. El único factor resultante (valoración de la educación física) lo componían 12 ítems, referidos a la valoración del profesorado hacia aspectos de la educación física que pueden tener influencia en las actividades acuáticas. Lo conformaban ítems como "Dos horas semanales de educación física no son suficientes para cumplir los objetivos que marca la legislación educativa" o "Los profesores de educación física no imparten aquellos contenidos que no saben o no controlan". La consistencia interna fue de .72. 
Albarracín, A.; Moreno, J. A. (2009). Problemática y beneficios de las actividades acuáticas en la escuela. Revista Internacional de Ciencias del Deporte, 16(5), 71-89. http://www.cafyd.com/REVISTA/01605.pdf

\section{Análisis descriptivo y de correlación del CAAEF}

Con respecto a las medias obtenidas (Tabla 3), el valor más alto lo presentaba el factor beneficios de la inclusión, muy seguido del factor problemática del profesorado. No se observó correlación significativa entre los dos factores.

Tabla 3. Media, Desviación Típica, Coeficiente Alfa y Correlaciones de Todas las Variables del CAAEF.

\begin{tabular}{|l|c|c|c|c|c|}
\hline & $M$ & $D T$ & $\alpha$ & Beneficios & Problemática \\
\hline Beneficios de la inclusión & 3.46 & .36 & .73 & - & .07 \\
\hline $\begin{array}{l}\text { Problemática del } \\
\text { profesorado }\end{array}$ & 3.25 & .86 & .74 & - & - \\
\hline
\end{tabular}

\section{Efectos principales y de interacción entre las variables}

Para comprobar los efectos principales se llevó a cabo una MANOVA, donde las variables independientes fueron el género, experiencia docente, titulación académica, cursos de formación, situación laboral, personal que ha impartido las clases cuando se han realizado actividades acuáticas en clases de educación física, y la valoración de aspectos destacables de educación física. Las variables dependientes fueron los factores beneficios de la inclusión y problemática del profesorado.

Se encontraron diferencias significativas (Tablas 4 y 5) en relación con el género $($ Lambda de Wilks $=.95, F(2,173)=4.43, p<.05)$, concretándose estas diferencias en los beneficios del medio acuático $(F=8.29, p<.05)$ con valores a favor de las mujeres frente a los hombres. La relación con el personal que impartía las clases presentó también diferencias significativas (Lambda de Wilks $=.87, F(4,142)=2.46, p<.05$ ) en el factor de problemática del profesorado. Tras el análisis post hoc (Tukey) éstas diferencias fueron a favor de aquellos docentes que habían impartido las clases conjuntamente con el técnico acuático, frente a los que las habían impartido solos y los que las habían dejado en manos exclusivamente del técnico acuático. En cuanto a la relación de la valoración de aspectos destacables de educación física con las actividades acuáticas educativas, se encontraron diferencias significativas (Lambda de Wilks $=.97$, $F(2,173), p<.05)$ en los beneficios, a favor de aquellos que menos valoraban estos aspectos frente a los que más los valoraban.

Tabla 4. Principales Efectos del Género, Experiencia, Titulación, Cursos, Situación, Personal que Imparte las Clases y la Valoración del Profesor Sobre Aspectos de Educación Física, sobre el CAAEF.

\begin{tabular}{|l|c|c|c|c|c|c|c|}
\hline Variables & Género & Experiencia & Titulación & Cursos & Situación & Personal & VEF \\
\hline & $F$ & $F$ & $F$ & $F$ & $F$ & $F$ & $F$ \\
\hline Beneficios & $8.29 *$ & .80 & .70 & 2.83 & .34 & .06 & 1.85 \\
\hline Problemática & .13 & .27 & 2.30 & 1.97 & 1.47 & $5.04^{*}$ & $3.91^{*}$ \\
\hline $\begin{array}{l}\text { Análisis } \\
\text { multivariado }\end{array}$ & & & & & & & \\
\hline Lambda de Wilks & .95 & .99 & .97 & .95 & .97 & .87 & .97 \\
\hline F multivariado & $4.43^{*}$ & .41 & 1.23 & 1.96 & 1.89 & $2.46^{*}$ & $2.17^{*}$ \\
\hline$* p<.05$
\end{tabular}


Albarracín, A.; Moreno, J. A. (2009). Problemática y beneficios de las actividades acuáticas en la escuela. Revista Internacional de Ciencias del Deporte, 16(5), 71-89. http://www.cafyd.com/REVISTA/01605.pdf

Tabla 5. Media y Desviación Típica por Género, Experiencia, Titulación, Cursos, Situación, Personal que Imparte las Clases y la Valoración del Profesor Sobre Aspectos de Educación Física.

\begin{tabular}{|c|c|c|c|c|c|c|}
\hline \multirow[t]{2}{*}{ Género } & \multicolumn{3}{|c|}{ Hombre $(n=129)$} & \multicolumn{3}{|c|}{ Mujer $(n=47)$} \\
\hline & \multicolumn{2}{|l|}{$M$} & $D T$ & \multicolumn{2}{|l|}{ M } & $D T$ \\
\hline Beneficios & \multicolumn{2}{|l|}{3.87} & .47 & \multicolumn{2}{|l|}{4.11} & .49 \\
\hline Problemática & \multicolumn{2}{|l|}{3.23} & .82 & \multicolumn{2}{|l|}{3.29} & .98 \\
\hline \multirow[t]{2}{*}{ Titulación } & \multicolumn{2}{|c|}{$\begin{array}{l}\text { Licenciado EF ( } n \\
=32)\end{array}$} & \multicolumn{2}{|c|}{ Diplomado EF $(n=17)$} & \multicolumn{2}{|c|}{ Otros $(n=27)$} \\
\hline & $M$ & $\overline{D T}$ & $M$ & $\overline{D T}$ & $M$ & $D T$ \\
\hline Beneficios & 3.92 & .46 & 4.02 & .67 & 4.02 & .50 \\
\hline Problemática & 3.18 & .85 & 3.62 & .93 & 3.38 & .83 \\
\hline \multirow[t]{2}{*}{ Situación } & \multicolumn{3}{|c|}{ Definitivo $(n=107)$} & \multicolumn{3}{|c|}{ Otros $(n=69)$} \\
\hline & \multicolumn{2}{|l|}{$M$} & $D T$ & \multicolumn{2}{|l|}{$M$} & $D T$ \\
\hline Beneficios & \multicolumn{2}{|l|}{3.38} & .49 & \multicolumn{2}{|l|}{4.02} & .48 \\
\hline Problemática & \multicolumn{2}{|l|}{3.18} & .77 & \multicolumn{2}{|l|}{3.35} & .98 \\
\hline \multirow[t]{2}{*}{ Tipo de cursos } & \multicolumn{2}{|c|}{$\begin{array}{l}\text { Ac. acuáticas ( } n \\
=20)\end{array}$} & \multicolumn{2}{|c|}{ Otros $(n=132)$} & \multicolumn{2}{|c|}{ Ninguno $(n=24)$} \\
\hline & $M$ & $D T$ & $M$ & $D T$ & $M$ & $\overline{D T}$ \\
\hline Beneficios & 4.18 & .52 & 3.92 & .48 & 3.87 & .47 \\
\hline Problemática & 3.49 & 1.04 & 3.27 & .78 & 2.98 & 1.06 \\
\hline \multirow[t]{2}{*}{ Experiencia } & \multicolumn{3}{|c|}{ Menos de 10 años $(n=83$} & \multicolumn{3}{|c|}{ Más de 10 años $(n=93)$} \\
\hline & \multicolumn{2}{|l|}{$M$} & $\overline{D T}$ & \multicolumn{2}{|l|}{ M } & $\overline{D T}$ \\
\hline Beneficios & \multicolumn{2}{|c|}{3.98} & .52 & 3.91 & & .46 \\
\hline Problemática & 3.29 & & .95 & 3.22 & & .78 \\
\hline Personal & Moni & $(n=25)$ & Profes & $=299$ & Mixta & $=21)$ \\
\hline & $M$ & $D T$ & $M$ & $D T$ & $M$ & $\overline{D T}$ \\
\hline Beneficios & 3.94 & .37 & 3.90 & .43 & 3.92 & .57 \\
\hline Problemática & 3.26 & .76 & 2.77 & .84 & 3.48 & .84 \\
\hline VEF & Deba & de la medi & $(n=9$ & Arriba c & medi & $=81)$ \\
\hline & $M$ & & $\overline{D T}$ & $M$ & & $\overline{D T}$ \\
\hline Beneficios & 3.99 & & .48 & 3.89 & & .49 \\
\hline Problemática & 3.37 & & .84 & 3.11 & & .87 \\
\hline
\end{tabular}

Respecto a los efectos de interacción (Tabla 6) se encontraron diferencias significativas $($ Lambda de Wilks $=.92, F(4,338)=2.62, p<.05)$ al relacionar la experiencia docente y la titulación del profesorado en el factor beneficios $(F=6.32, p<.05)$. De igual forma, en cuanto a la relación entre la experiencia docente y los cursos realizados, también se encontraron diferencias significativas (Lambda de Wilks $=.94, F(4,338)=$ $3.56, p<.05)$ con respecto a la dimensión problemática del profesorado $(F=5.00, p<$ $.05)$. La relación entre la titulación del profesorado y el género también presentó diferencias significativas (Lambda de Wilks $=.93, F(4,338)=2.71, p<.05$ ) en relación a ambos factores, tanto al de beneficios $(F=4.32, p<.05)$, como al de problemática del 
profesorado $(F=3.04, p<.05)$. Al relacionar la titulación con el tipo de cursos que ha realizado el docente, las diferencias significativas (Lambda de Wilks $=.89, F(8,332)=$ $2.34, p<.05)$ aparecieron en los beneficios $(F=4.05, p<.05)$. También se encontraron diferencias significativas en la interacción de la titulación y la situación administrativa del docente (Lambda de Wilks $=.93, F(4,338)=2.76, p<.05$ ) en el factor problemática del profesorado $(F=4.83, p<.05)$. Por último, al relacionar la situación administrativa con los cursos realizados también se encontraron diferencias significativas $($ Lambda de Wilks $=.93, F(4,338)=3.11, p<.05)$ en la problemática del profesorado $(F=6.15, p<.05)$.

Tabla 6. Principales Efectos de Interacción Según el Género, Experiencia, Titulación, Cursos y Situación Laboral sobre el CAAEF.

\begin{tabular}{|l|c|c|c|c|c|c|}
\hline Variables & $\begin{array}{c}\text { Experiencia } \\
\text { x titulación }\end{array}$ & $\begin{array}{c}\text { Experiencia } \\
\text { x cursos }\end{array}$ & $\begin{array}{c}\text { Titulación } \\
\text { x género }\end{array}$ & $\begin{array}{c}\text { Titulación } \\
\text { x cursos }\end{array}$ & $\begin{array}{c}\text { Titulación } \\
\text { x } \\
\text { situación }\end{array}$ & $\begin{array}{c}\text { Cursos x } \\
\text { situación }\end{array}$ \\
\hline Beneficios & $F$ & $F$ & $F$ & $F$ & $F$ & $F$ \\
\hline Problemática & $6.32^{*}$ & 1.26 & $4.32^{*}$ & $4.05^{*}$ & 2.48 & .25 \\
\hline Análisis multivariado & 2.00 & $5.00^{*}$ & $3.04^{*}$ & 1.87 & $4.83^{*}$ & $6.15^{*}$ \\
\hline Lambda de Wilks & .92 & .94 & .93 & .89 & .93 & .93 \\
\hline F multivariado & $3.56^{*}$ & $2.62^{*}$ & $2.71^{*}$ & $2.34^{*}$ & $2.76^{*}$ & $3.11^{*}$ \\
\hline
\end{tabular}

\section{Discusión}

El objetivo del estudio ha consistido en comprobar la importancia que las actividades acuáticas tienen en el marco de la educación física en Educación Secundaria para lo que se ha tenido que diseñar y validar el Cuestionario Actividades Acuáticas en Educación Física (CAAEF) y la Escala de Valoración de la Educación Física (VEF). También se han comprobado los efectos de interacción del género del profesorado, experiencia docente, titulación, tipo de cursos realizados, situación administrativa, quién ha impartido las sesiones acuáticas y la valoración sobre aspectos de educación física que pueden influir en la inclusión de las actividades acuáticas en dicha materia.

Para determinar la estructura factorial de los instrumentos se ha realizado un análisis factorial exploratorio, $y$ se ha obtenido en el caso del CAAEF una escala compuesta por 14 ítems divididos en dos factores: beneficios de la inclusión y problemáticas presentadas a los docentes, con una varianza total explicada de $35.98 \%$. Se han obtenido resultados satisfactorios en la consistencia interna. El factor referido a los beneficios, consta de ítems que aluden a aspectos tales como las ventajas del medio acuático sobre el terrestre en cuanto a lesiones, desarrollo motor, seguridad de accidentes, motivación hacia el medio, actividad física recomendada, falta de práctica habitual del alumnado, etc. El factor acerca de la problemática se refiere principalmente a la formación de los docentes en actividades acuáticas, así como a la seguridad de los mismos en dichas salidas a las instalaciones acuáticas. En el caso del VEF, con 12 ítems unificados en un solo factor, también ha obtenido resultados satisfactorios de consistencia interna, con una varianza total explicada del $13.93 \%$. De este modo, se presentan los instrumentos CAAEF y VEF como herramientas válidas y fiables para medir aspectos relacionados con las actividades acuáticas educativas en educación física, valorando tanto los 
beneficios como la problemática de aplicación de las mismas, si bien es necesario realizar más investigaciones que confirmen los índices aquí obtenidos.

En relación al género, las profesoras son las que más valoran los beneficios del medio acuático dentro de la materia de educación física. Por otro lado, al relacionar los aspectos de las actividades acuáticas con el personal que ha impartido las clases en el caso de haber podido llevarlas a la práctica en su labor docente, resulta que los docentes que han dirigido personalmente son los que menos valoran la problemática de la aplicación de estos contenidos. Es posible que esto se deba a que aquellos que consideran las actividades acuáticas como un contenido más dentro de su programación, y como tal deben impartirlo, no encuentran dificultades en la falta de formación ni en el desconocimiento de la aplicación de dichas actividades. Seguidamente se encuentran los que han dejado en manos del técnico acuático sus clases, pudiendo significar esto una mayor despreocupación o desinterés por esta tarea. Es interesante observar cómo aquellos que han demandado la ayuda o colaboración del técnico acuático para impartir sus clases son los que más consideran esta problemática, siendo ellos los que entienden que existe desconocimiento de la aplicación de estos contenidos, así como la falta de formación al respecto, razón por la cual deben solicitar apoyo de otro profesional.

En la bibliografía al respecto se encuentran posturas opuestas, y sería preciso distinguir si nos referimos a una situación ideal o a la realidad del momento. Así, en búsqueda de una aplicación correcta y adecuada, Domínguez (1989) no duda de que estas clases, puesto que si forman parte de la materia de educación física, deben ser impartidas por los docentes de la misma, aunque siempre con la ayuda de un técnico. En cambio, otros autores (Albarracín, 2007; Rodríguez y Moreno, 1997) insisten en que debe ser sólo el docente el encargado de tal labor, igual que ocurre con el resto de contenidos. Frente a todo ello, se reconoce que la realidad difiere de todo el planteamiento anterior, ya que la gran parte de los programas escolares son impartidos por técnicos de la instalación (López y Aznar, 2003; Tuero y Salguero, 2003). Esta falta de participación de los docentes de educación física se puede deber, según estudios de Gadd y Hanbury (2004) a la falta de seguridad en la materia, teniendo como referencia que en Enseñanza Secundaria en Gales, un 24.4\% solicitaba ayuda del técnico por esta circunstancia. En Francia en cambio, poseen un proceso de formación específico existiendo como resultado docentes especialistas con acreditación en la materia (Reeves, 2001). De nuevo, toda esta reflexión no debe desvincularse de la formación de unos y otros respecto de la materia de educación física y de la tendencia de su formación acuática.

Si concretamente incidimos en la formación del docente responsable, habría que distinguir entre su formación académica (titulación) y la continua (cursos de actualización). En cuanto a la académica, parece que el ser Licenciado en Ciencias de la Actividad Física y del Deporte ${ }^{2}$, no implica ni considerar más los beneficios del medio acuático en el ámbito escolar, ni ver disminuida la problemática al aplicar estos contenidos. Muy al contrario, son tanto los Diplomados en Educación Física como otros titulados los que consideran más dichos beneficios y otorgan más importancia a las dificultades que afrontan los docentes. Sí que es cierto que entre estas dificultades, las principales están enfocadas a la formación en estos contenidos, por lo que quizá no encuentren en dicho aspecto una traba para ser aplicados curricularmente con normalidad. Por otra parte, si nos centramos en el tipo de cursos realizados, es decir, en la formación continua del docente, entonces sí que se encuentran diferencias a favor de

\footnotetext{
${ }^{2}$ Recordemos la obligatoriedad de la asignatura en las Facultades de Ciencias de la Actividad Física y el Deporte durante muchos años.
} 
los que se han centrado en cursos de actividades acuáticas, ya que son éstos los que más consideran los beneficios de dichas tareas curriculares, y además son los que más problemática advierten a la hora de aplicarlos en sus clases de educación física.

En este sentido se da a entender que parte de las reticencias a la inclusión de las actividades acuáticas se centra en la formación del profesorado, tanto la inicial como la permanente, que según Rodado (1992) han adolecido de los mismos errores, ya que no se sustentan en una base de reflexión e intervención pedagógicas del docente en activo, pues son éstos los que deben determinar la dirección y modalidades de su desarrollo personal y profesional. Por eso, sí es cierto que se deben presentar formas flexibles e innovadoras que den respuesta a los cambios del sistema educativo, los responsables de la formación deben integrarse en el sistema y adaptarse a las demandas y necesidades concretas. Así, no basta con la motivación del docente que elimine su práctica "rutinaria", sino que desde este punto de vista la innovación metodológica debe plantearse ayudas innovadoras, aportar medios de acceso a las nuevas tecnologías, etc., no quedándose en una declaración de intenciones. Así se solucionaría, y según el citado autor, no sólo la intervención didáctica, sino el estatus social tan deteriorado por este sector educativo (en especial en esta disciplina). De esta forma se llegaría a unir dichas innovaciones con los problemas reales que el docente encuentra en sus clases a diario, y siendo la base para la formación del docente, tanto la permanente como la inicial, incluyendo en el proceso también a los formadores de enseñantes ${ }^{3}$.

En definitiva, podemos afirmar que la formación en determinada materia va a influir decisivamente en la impartición de esos contenidos por parte del profesorado, y más aún en algunos de ellos que no son muy habituales. Además, esta situación no es específica de las actividades acuáticas, ya que varios autores (Archilla y Pérez, 2003; Machota, 2004; Sánchez y Coterón, 2007; Vaquero, 2003) alegan las mismas razones a la ausencia de expresión corporal en la aplicación real de las programaciones didácticas. Lo único que nos queda es pensar que la formación en actividades acuáticas está creciendo, así como la concienciación de su importancia, por lo que la tendencia debería ser su inclusión definitiva en los currículos escolares.

Al tener en cuenta ciertos aspectos importantes de educación física que podrían tener repercusión en la aplicación concreta de las actividades acuáticas, aquellos que más han considerado estos aspectos generales de la materia, son los que menos están de acuerdo con la problemática concreta de la realización de contenidos en el medio acuático. De esta forma, estos docentes consideran más importantes la situación general de educación física que la concreta de los contenidos acuáticos, considerando que afectan más determinados aspectos como el peso curricular de la materia dentro del currículo, la sensibilización de los padres y compañeros hacia la materia, no impartir por parte de los docentes aquellos contenidos que no se dominan, etc. Lo cierto es que se ha tratado el bajo estatus que acecha esta materia en los últimos tiempos (Kirk, 1990; Vizuete, 1994), habiendo sido señalado en diferentes investigaciones centradas en la opinión de los docentes (Acha, Velásquez, y Moya, 2007; Machota, 2004, entre otros), debiendo según ellos solucionar esta situación para poder afrontar con mayor seriedad la labor docente por medio de unas programaciones adecuadas. Igualmente es señalado en varios estudios (Acha et al., 2007; Siedentop, 1998) la baja consideración de la educación física por parte de los compañeros de otras materias, llegando a hablar incluso de discriminación de la misma. Esto sugiere que se considere que para llevar a cabo unas

\footnotetext{
3 Así se relaciona la labor docente con las necesidades formativas en la Universidad, no siendo estamentos diferenciados, sino reconociendo la continuidad de ambos.
} 
prácticas curriculares como las tratadas aquí se precisa una colaboración con todos los miembros del claustro, así como principalmente con la dirección del centro, para solucionar aspectos como horarios, salidas del centro a la instalación acuática, etc. (Bass y Hardy, 1997). Por tanto, parece que aquellos que más consideran estas circunstancias, suponen que las específicas del ámbito acuático son secundarias con respecto a éstas, ya que las primeras imposibilitan más que las segundas. Entre otras circunstancias que ineludiblemente afectan a la aplicación de un contenido como es el acuático, con más demanda de requerimientos en cuanto al tiempo, resulta decisiva la escasez de horas de que dispone esta materia escolar (Acha et al., 2007; Machota, 2004; Pieron, 2007), siendo en muchos casos la causa de la imposibilidad de aplicadar unas actividades como las acuáticas.

Consideramos que la ausencia de referencias legales hacia estos contenidos explican buena parte de todas estas circunstancias, por lo que como ya se indicaba años atrás (Moreno, 2000) creemos necesario que debería de hacerse eco de las nuevas perspectivas curriculares que insisten en lograr el desarrollo integral de la persona a través de la utilización de todos los medios disponibles al alcance del docente, siendo uno de ellos el medio acuático. De esta manera, se podrá conseguir que las actividades acuáticas y la natación no sean algo aislado ni ajeno a la educación del alumnado.

\section{Conclusiones}

Confirmando nuestra hipótesis, los docentes que consideran en menor grado ciertos aspectos negativos de educación física, son los que más pueden considerar los beneficios de la inclusión de las actividades acuáticas. De igual forma, el personal que ha llevado a cabo las clases será decisivo a la hora de considerar más o menos problemática en la aplicación de las actividades acuáticas. Sin embargo, no confirma nuestra hipótesis los resultados con respecto al género, puesto que aunque nos parece que todos los docentes deben considerar los mismos beneficios de estos contenidos, en este caso, son las mujeres las que han destacado su inclusión en educación física.

\section{Contribución académica y futuras líneas de investigación}

La contribución académica de estos resultados está encaminada no tanto a la aportación de materiales y soluciones, sino a la reflexión de la necesidad de dar a conocer a los docentes de educación física los beneficios de las actividades realizadas en el medio acuático, tanto por razones educativas (mejora del desarrollo motriz y psicológico, participación de exentos, conocimiento de nuevas actividades físicas y participación de todos los bloques de contenidos), como metodológicas (motivación y atracción del medio, diversión, etc.) y utilitarias (evitar accidentes y practicar una actividad muy prescrita por los médicos). Por otra parte, contribuye a especificar los problemas que los docentes encuentran al aplicar estos contenidos, o simplemente al intentarlo, para a partir de ello intentar subsanarlos, puesto que se destaca principalmente la formación en dicha materia, debiendo de intervenir tanto los responsables de la formación inicial en el ámbito universitario como de la continua en el ámbito educativo y escolar.

En cuanto a futuras líneas de investigación que se derivan de este estudio, sería importante profundizar en el conocimiento concreto de las carencias de los docentes, analizando cada una de las razones que conducen a no llevar a cabo estos contenidos así como no ser los responsables de las mismas una vez puestas en práctica. También sería interesante el estudio del procedimiento adecuado de los docentes de la materia en este ámbito, analizando estrategias y programas de promoción de dichas actividades dentro del ámbito escolar según la importancia que se le atribuya a éste (Albarracín, 2005, 
2007; Cotrino, Moreno, y Pérez, 2005; Marín, Zomeño, Martínez, Alonso, y GonzálezCutre, 2008; Moreno y Gutiérrez, 1998; Muñoz, 2004; Prieto y Nistal, 2001, 2005). En concreto, algunas son las soluciones que se han presentado en algunos estudios, pudiendo analizar el grado que cada una de ellas presenta en la mejora de la situación actual ya señalada en este trabajo.

\section{Referencias}

Acha, A.; Velásquez, R., y Moya, J. M. (2007). El estatus de la educación física en el currículum de Educación Secundaria Obligatoria. Algunas propuestas de solución. En Actas de II Congreso Internacional y XXIV Nacional de Educación Física. Palma de Mallorca: Universidad de Islas Baleares.

Albarracín, A. (2003). Los libros de texto referidos al medio acuático. En Actas del Congreso Mundial de Ciencias de la Actividad Física y el Deporte (p. 195). Granada: Universidad de Granada.

Albarracín, A. (2005). Las Actividades Acuáticas en los libros de texto de Educación Física según los profesores. En J. A. Moreno (Ed). II Congreso Internacional de Actividades Acuáticas (pp. 212-225). Murcia: Universidad de Murcia.

Albarracín, A. (2007). El medio acuático y sus posibilidades de acción. Propuesta de inclusión de las actividades acuáticas en los diferentes niveles de Educación Física en Educación Secundaria. En Actas de II Congreso Internacional y XXIV Nacional de Educación Física. Palma de Mallorca: Universidad de Islas Baleares.

Archilla, M. T., y Pérez, D. (2003). Los conflictos y problemas a los que nos enfrentamos el profesorado a la hora de desarrollar actividades expresivas de educación física. En G. Sánchez, B. Tabernero, F. J. Coterón, C. Llanos, y B. Learreta (Eds). Expresión, creatividad y movimiento. I Congreso Internacional de Expresión Corporal y Educación (pp. 333-338). Salamanca: Amarú.

Barter, T. J.; Firth, C. (1994). The National Curriculum- Swimming. The British Journal of Physical Education, 3, autumn, 17-19.

Bass, D., y Hardy, C. A. (1997). That sinking feelin: Swimming in Primary Schools Post the 1988 Education Reform Act. European Journal of Physical Education, 2, 178-197.

Benito, J. (1995). La Educación parea la paz como materia transversal. Tesis Doctoral. Murcia: Universidad de Murcia. Facultad de Educación.

Blández, J. (1996). La investigación- acción: un reto para el profesorado. Barcelona: Inde.

Boixeda, A. (2003). La motricidad humana: un planteamiento para su desarrollo en el medio acuático, en etapa escolar. I Congreso Internacional de Actividades Acuáticas. Murcia: ICD.

Casterad, J. (2003). Nuevas posibilidades curriculares para la natación educativa. En F. Ruiz, y E. P. González del Hoyo (Eds), Educación física y deporte en edad escolar. Actas del $V$ Congreso Internacional de FEADEF (pp. 505-509). Valladolid: Diputación de Valladolid.

Casterad, J. (2004). La formación continua de técnicos municipales de natación. En Actas del XXII Congreso Nacional de Educación Física: "La formación inicial del profesorado de Educación Física ante el reto europeo. La Coruña: Universidad de La Coruña.

Cotrino, J. A.; Moreno, J., y Pérez, J. A. (2005). La educación física en el medio acuático. Sevilla: Wanceulen. 
Decreto 112/2002, de 13 de septiembre por el que se establece el currículo de la Educación Secundaria Obligatoria en la Comunidad Autónoma de la Región de Murcia. BORM no 214 de 14 de septiembre de 2002

Decreto $113 / 2002$, de 13 de septiembre por el que se establece el currículo del Bachillerato en la Comunidad Autónoma de la Región de Murcia. BORM no 214 de 14 de septiembre de 2002.

Decreto 291/2007, de 14 de septiembre, por el que se establece el currículo de la Secundaria Obligatoria en la Comunidad Autónoma de la Región de Murcia. BORM n० 221 de septiembre de 2007.

Decreto 262/2008, de 5 de septiembre por el que se establece el currículo del Bachillerato en la Comunidad Autónoma de la Región de Murcia. BORM no 211 de 10 de septiembre de 2008.

Del Castillo, M. (2001). Motricidad acuática e iniciación deportiva. NSW, XXIII (3), 26-34.

Domínguez, F. (1989). La natación a través de un programa de Educación Física Escolar. NSW, 11(2), 21-40.

Gadd, J., y Hanbury, A. (2004). Physical education and school sport project in Wales. Continuous professional development survey. The British Journal of Teaching Physical Education, 3, 23-26.

González, C.; Herrera, V.; Palmero, A.; Hernando, A.; Sullana, T.; Cardona, M., y Barceló, G. (2003). Zambúllete en la piscina, lánzate al agua: dos propuestas formativas para el área de Educación Física. En Actas del I Congreso Internacional de Actividades Acuáticas. Murcia: ICD.

Gourson-Verger, N., y Verger, M. (2003). Apprentissage ludique des non-nageurs. EPS, 304, 39-46.

Hardman, K. (2001). The world-wide survey of Physical education in schools: findings, issues and strategies for a sustainable future. The British journal of teaching Physical Education, 1, 29-31.

Jofre, E., y Lizalde, M. (2003). Las actividades acuáticas en los currículos de educación física en la etapa de primaria. En Actas I Congreso Internacional de Actividades Acuáticas. Murcia: ICD.

Joven, A. (1990). Realidad y expectativas de la natación educativa. Una aproximación práctica. Apunts: Educació Física i Esports, 21, 11-16.

Joven, A. (2001a). Estudio y evolución de las actividades acuáticas: La formación de técnicos. Tesis Doctoral. Barcelona: Universitat de Barcelona. Departament de Teoria i Història de I'Educació.

Joven, A. (2001b). La natación hoy. Evolución de los programas acuáticos en los últimos años. Comunicaciones Técnicas, 3, 3-14.

Kirk, D. (1990). Educación física y currículum: introducción crítica. Valencia: Universidad de Valencia. Servicio de Publicaciones.

Lacasa, A., y López, J. (2002). Una unidad didáctica diferente... iconoce la piscina! Comunicaciones Técnicas, 2, 53-60.

Lawton, J., y Lee, C. (1995). Swimming in the National Curriculum. The British Journal of Physical Education, 4, 14-15.

Le Boulch, J. (1991). El deporte educativo. Psicocinética y aprendizaje motor. Barcelona: Paidós. 
López, E., y Aznar, N. (2003). Las actividades acuáticas educativas y recreativas en la formación del maestro. En Actas del $V$ Congreso Internacional de FCADEF "Educación física y deporte en edad escolar" (pp. 467-471). Valladolid: Diputación de Valladolid.

Lynn, M. (1986). Determination and quantification of content validity. Nursing Research, 35, 382-385.

Machota, V. E. (2004). Del diseño al desarrollo curricular de la educación física en la Enseñanza Secundaria Obligatoria: entre las intenciones y la práctica. Tesis Doctoral. Madrid: Universidad Politécnica de Madrid.

Marín, L. M.; Zomeño, T.; Martínez, C.; Alonso, N., y González-Cutre, D. (2008). Actividades acuáticas como parte del currículum de Enseñanza Secundaria y Bachillerato. En J. A. Moreno y L. M. Marín (Eds). Nuevas aportaciones a las actividades acuáticas ( $p p$. 54-68). Murcia: Unidad de Investigación en Educación Física y Deportes.

Miró, P. (1984). Les Activitats Aquatiques en el programa d'Educació Física. Apunts, $X X I, 29-35$.

Moreno, J. A. (2000). El juego acuático como medio de desarrollo de las actividades acuáticas en la escuela. En Actas de Cursos de Verano Universidad del Mar. Murcia: Universidad de Murcia.

Moreno, J. A. (1998). Relación oferta- demanda de las instalaciones acuáticas cubiertas: bases para un programa motor en actividades acuáticas educativas. Facultad de Psicología. Tesis Doctoral. Universidad de Valencia.

Moreno, J. A., y Gutiérrez, M. (1998). Bases metodológicas para el aprendizaje de las actividades acuáticas educativas. Barcelona: Inde.

Muñoz, A. (2004). Actividades acuáticas como contenido del área de Educación Física. Lecturas: Educación Física y Deportes. Revista Digital, 73, Año 10. $<A$ :/Actividades acuáticas como contenido del área de Educación Física.htm> [Consulta: 26/10/2004].

Navarro, F. (1990). Hacia el dominio de la natación. Madrid: Gymnos.

Osterlind, S. J. (1989). Constructing Test Items. Londres: Kluwer Academic Publishers.

Pérez , B. (1998). Natación Infantil Argentina. La historia educativa que me tocó vivir. Buenos Aires: Lecturas: Educación física y deportes. Revista Digital, 11, Año 3. <http://www.efdeportes.com/efd11/bperez.htm > [Consulta: 29/4/2000].

Pichot, D. (2006). Las reglas institucionales para realizar una enseñanza eficaz de la natación en Francia. Cursos de Verano de la Universidad de Palencia. Palencia: Universidad de Palencia.

Pieron, M. (2007). Educar para un estilo saludable: un desafío para la escuela y los profesores de educación física. En P. Palou, J. Ponseti, P. A. Borrás, y J. Vidal (Eds). Educación Física en el siglo XXI. Nuevas perspectivas. Nuevos retos (pp. 33-64). Palma de Mallorca: Universidad de Islas Baleares.

Prieto, J. A., y Nistal, P. (2001). El medio acuático en el área de la educación física. Lecturas: Educación Física y Deportes. Revista Digital, 42, Año 7. <http://www. efdeportes.com/efd42/acuat.htm >. [Consulta: 7/9/2002].

Rédaction EPS 1 (2003). Natation scolaire. Un nouveau cadre d'enseignement. EPS $1,114,9-10$.

Reeves, B. (2001). Whiter Physical Education? The British Journal of Teaching Physical Education, 32 (1), 48-50. 
Rodado, P. (1992). Reflexiones en torno a la formación permanente del profesorado. Habilidad Motriz, 0, 20-22.

Rodríguez, P. L, y Moreno, J. A. (1997). Un modelo de actuación para actividades acuáticas en primaria. Habilidad Motriz, 10, 38-49.

Sánchez, G., y Coterón, J. (2007). Expresión corporal en educación física: un costoso entendimiento. En Actas de II Congreso Internacional y XXIV Nacional de Educación Física. Palma de Mallorca: Universidad de Islas Baleares.

Siedentop, D. (1998). Aprender a enseñar la educación física. Barcelona: Inde.

Stevens, J. (1992). Applied multivariate statistics for the social sciences. Hillsdale, $\mathrm{NJ}$ : Lawrence Erlbaum.

Terret, T. (1998). L'eau, l'école et l'espace. Normes scolaires et pratiques de la natation au XXe siècle. En P. Arnaud y T. Terret (Eds). Le sport et ses espaces. XIXe- XXe siècles (pp. 47-63). París: CTHS.

Tuero, C., y Salguero, A. (2003). Las actividades en el medio acuático. En B. Tabernero (Ed). Educación física: propuestas para el cambio (pp. 33-64). Barcelona: Paidotribo.

Vaca, M. J. (2000). La enseñanza de la natación escolar en Palencia. En Actas del I Congreso Nacional de Natación Escolar. Cuadernos Técnicos (pp. 19-36). Palencia: Patronato Municipal de Deportes. Ayuntamiento de Palencia.

Vaquero, A. (2003). La presencia de los propósitos educativos vinculados a la dimensión expresiva del movimiento en educación primaria. En G. Sánchez, B. Tabernero, F. J. Coterón, C. Llanos, y B. Learreta (Eds). Expresión, creatividad y movimiento. I Congreso Internacional de Expresión Corporal y Educación (pp. 339-344). Salamanca: Amarú.

Vizuete, M. (1994). La identidad de la educación física en un contexto europeo. Habilidad motriz, 5, 13-19.

Wigmore, S. (1994). Swimming- an activity for live? Article $n^{0} 1$ in a series of 3 articles. The Bulletin of Physical Education, 30 (3), 35-42.

Wigmore, S. (1995). Swimming on prescription. Article no 2 in a series of 3 articles. The Bulletin of Physical Education, 31 (3), 22-25.

Wigmore, S. (1997). Swimming- its educational value. Article $n^{\circ} 3$ in a series of 3 articles. The Bulletin of Physical Education, 33 (2), 6-9.

Williams, A.; Bedward, J., y Woodhouse, J. (2000). An inclusive National Curriculum? The experience of adolescent girls. European Journal of Physical Education, 5 (1), 4-18.

Zwozdiak-Myers, P. (2004). Breadth, Balance and Relevance! A report of year 6 and year 7 pupils' curricular and extra-curricular experiences in the East of England. The British Journal of Teaching Physical Education, 2, 43-49. 


\section{Anexo}

1. Nombre del centro

2. Localidad.

3. El centro es:

o Público

○ Privado

4. Situación administrativa:
o Profesor/a definitivo/a
- Interino/a

o Profesor/a provisional

○ Otro

5. Edad:

o Entre 21 y 30 años

$\circ$ Entre 31 y 40 años

○ Entre 41 y 50 años

6. Género:

$$
\text { ○ Hombre }
$$

7. Titulación:

o Diplomado/a en educación física

- Diplomado/a en otra especialidad

o Licenciado/a en educación física

8. Su experiencia docente es de:
- Menos de 5 años
○ Entre 6 y 10 años
○ Entre 11 y 15 años

Entre 51 y 60 años

Más de 60 años

○ Mujer

- Licenciado/a en otra especialidad

- Catedrático/a en educación física

- Otros

- Entre 16 y 20 años

- Más de 20 años

9. ¿Qué tipo de cursos de actualización has realizado en educación física?
○ Educación física general
○ Legislación
○ Expresión corporal
○ Deportes
- Actividades en naturaleza
- Actividades acuáticas

10. ¿Tienes alguna instalación cercana o disponible adecuada para llevar a cabo un programa de actividades acuáticas o la has tenido en algún curso escolar?

11. ¿Has llevado a tus alumnos a la piscina en horario lectivo en algún curso escolar?

12. En caso afirmativo, ¿qué personal impartió las clases?
- El monitor
- Yo como profesor de educación física
- Los dos a la vez

13. A continuación te presentamos una serie de cuestiones que te pedimos que señales el grado de conformidad o disconformidad:

1. El término "actividades acuáticas educativas" es un gran desconocido por los profesores de educación física

2. Los beneficios físicos y psíquicos que proporciona el medio acuático por sus características especiales son mayores que en el medio terrestre

3. La actividad física en el medio acuático tiene menos peligro para las lesiones (menos impacto, más relajación) por lo que es muy beneficiosa

4. Todos los bloques de contenido de la educación física son susceptibles de ser impartidos en el agua, con la adecuación suficiente

5. El desarrollo motriz en el niño se ve favorecido por una experiencia precoz en el medio acuático

6. Es importante que todos los niños en edad escolar sepan nadar para evitar

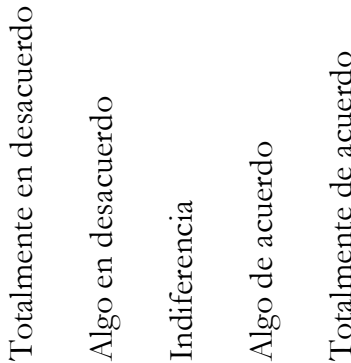

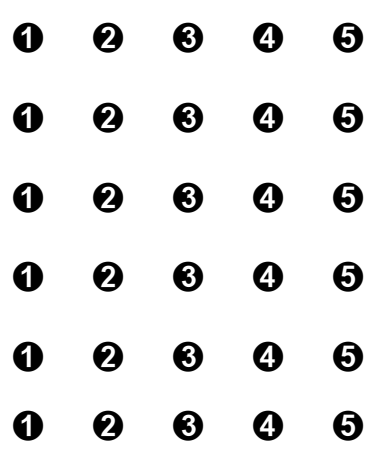


accidentes

7. Las actividades acuáticas no se introducen habitualmente en las programaciones de educación física por falta de instalaciones

8. Las actividades acuáticas no se introducen habitualmente en las programaciones de educación física porque el profesorado no están formados para ello

9. La natación es un deporte conocido, practicado y beneficioso como para se incluido en las clases de educación física

10. La natación es el deporte más completo y el más prescrito por los médicos

11. En una clase de educación física en el agua conseguiríamos la participación de los alumnos exentos por problemas de espalda

12. El medio acuático es atractivo, motivante y novedoso, y el aspecto recreativo lo tenemos asegurado

13. Muchos alumnos sólo han asistido a la piscina para bañarse, y desconocen otras posibles actuaciones

14. Mucho profesorado de educación física desconocen las posibles aplicaciones de las Actividades acuáticas en su materia

15. Mucho profesorado de educación física, aún teniendo posibilidad de ir a piscinas, no lo hacen por comodidad y seguridad personal

16. Me parecería adecuado una legislación que obligase a impartir actividades acuáticas a ciertos niveles, facilitando el tema de instalación y seguridad

17. En otros países la legislación educativa facilita ciertos contenidos (esquí, natación, orientación, etc.), y se imparten de forma cotidiana dentro del currículo ordinario

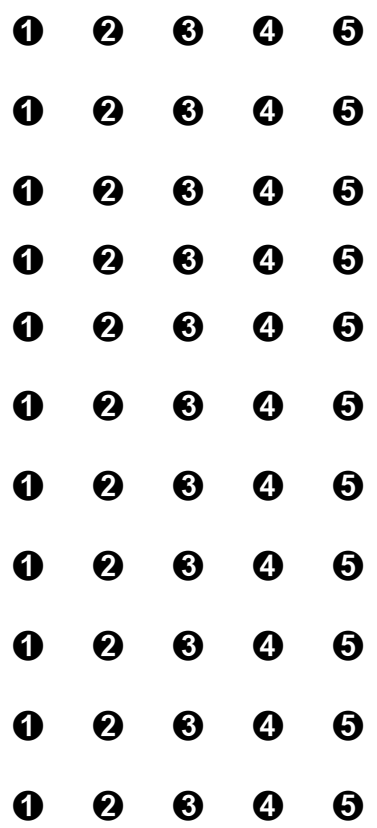

\section{Otras cuestiones que pueden relacionar la educación física con actividades acuáticas}

1. Creo que los compañeros de otras materias no valoran demasiado a la educación física

2. En general, los padres están convencidos de que la educación física no tiene entidad propia.

3. Aún hoy, uno de los trabajos más laboriosos es sensibilizar a los padres sobre la importancia de la educación física

4. Los centros educativos no están acondicionados para ofrecer una educación física de calidad

5. Para educar como pretende la Ley, el profesorado debería tener un máximo de 20 alumnos por grupo

6. Me preocupa no disponer de conocimientos y estrategias que orienten mi práctica educativa hacia la legislación educativa actual

7. La educación física sigue siendo considerada como una materia complementaria

8. La educación física debería tener mayor peso específico en el currículo

9. Dos horas semanales de educación física no son suficientes para cumplir los objetivos que marca la Legislación educativa.

10. Los profesores de educación física no imparten aquellos contenidos que no saben o no dominan

11. Es importante cumplir las prescripciones de la Ley acerca de aumentar el número de experiencias motrices

12. En educación física se consideran peligrosas ciertas innovaciones curriculares, por lo que no se llevan a cabo

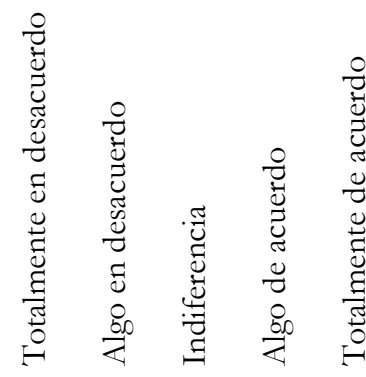

$\begin{array}{lllll}1 & 2 & 3 & 4 & 5 \\ 1 & 2 & 3 & 4 & 5\end{array}$

$\begin{array}{lllll}1 & 2 & 3 & 4 & 5\end{array}$

(1) 2030

$\begin{array}{lllll}1 & 2 & 3 & 4 & 5\end{array}$

$\begin{array}{lllll}1 & 2 & 3 & 4 & 5\end{array}$

$\begin{array}{lllll}1 & 2 & 3 & 4 & 5\end{array}$

$\begin{array}{lllll}1 & 2 & 3 & 4 & 5\end{array}$

$\begin{array}{lllll}1 & 2 & 3 & 4 & 5\end{array}$

$\begin{array}{lllll}1 & 2 & 3 & 4 & 5\end{array}$

$\begin{array}{lllll}1 & 2 & 3 & 4 & 5\end{array}$

$\begin{array}{lllll}1 & 2 & 3 & 4 & 5\end{array}$ 